\title{
APPARATUS FOR THE ANALYSIS OF ILLUMINATING AND FUEL GASES.
}

\author{
By Gro. E. THOMAs. \\ Rereived Juiy 2x, 1809.
}

TH

EERE are many difficulties in the manipulation of the various forms of apparatus now employed for the analysis of illuminating and fuel gases. Much danger of loss is incurred in some methods of procedure incident to the repeated connecting and disconnecting of apparatus, while the size of others renders them inapplicable for easy transportation; also the necessity of frequent cleansing in some forms and the fragility of the material due to peculiarities of construction offer many obstacles in this line of chemical analysis. With these objectionable features in mind and with the desire to construct a piece of apparatus compact and efficient for technical work, the production of the form represented by figure I was undertaken, believing that there would be a demand for it.

The apparatus here described consists of a burette and leveling tube joined by rubber and capillary glass tubing with bottles, each of which is provided with a funnel. The dimensions are sixteen inches long by fifteen inches high. The burette is graduated to Ioo cc., forty in whole dimensions, represented by the bulb 3, and the remainder in fifths of the same and is supplied with platinum terminals 4 and 5 , about one-half inch from the top; it is connected. with the leveling tube 7 by rubber tubing and is filled with water acidulated with sulphuric acid to decrease the solubility of the carbon dioxide in the water. The burette is joined to the absorbing bottles I5, I6, and I 7 and the storing bottle I 8 by the capillary tube $e$ and the capillary $T$ tubes I I, I 2, I 3 , and I4 having stop-cocks I9, 20, 2 I, and 22 connected by rubber tubing; 14 is provided with a plug 34 . The funnel tube of each bottle serves for the exit of liquid from below. Tubes 23,24 , and 25 are supplied with stop-cocks $b, c$, and $d$, none being required on the last tube as bottle 8 is used only for storing. Bottle 5 is filled with a solution of caustic potash; larger surface to increase the absorption is obtained by the use of glass tubes. Bottle 6 contains water saturated with bromine, while 17 holds the phosphorus covered with water, being 


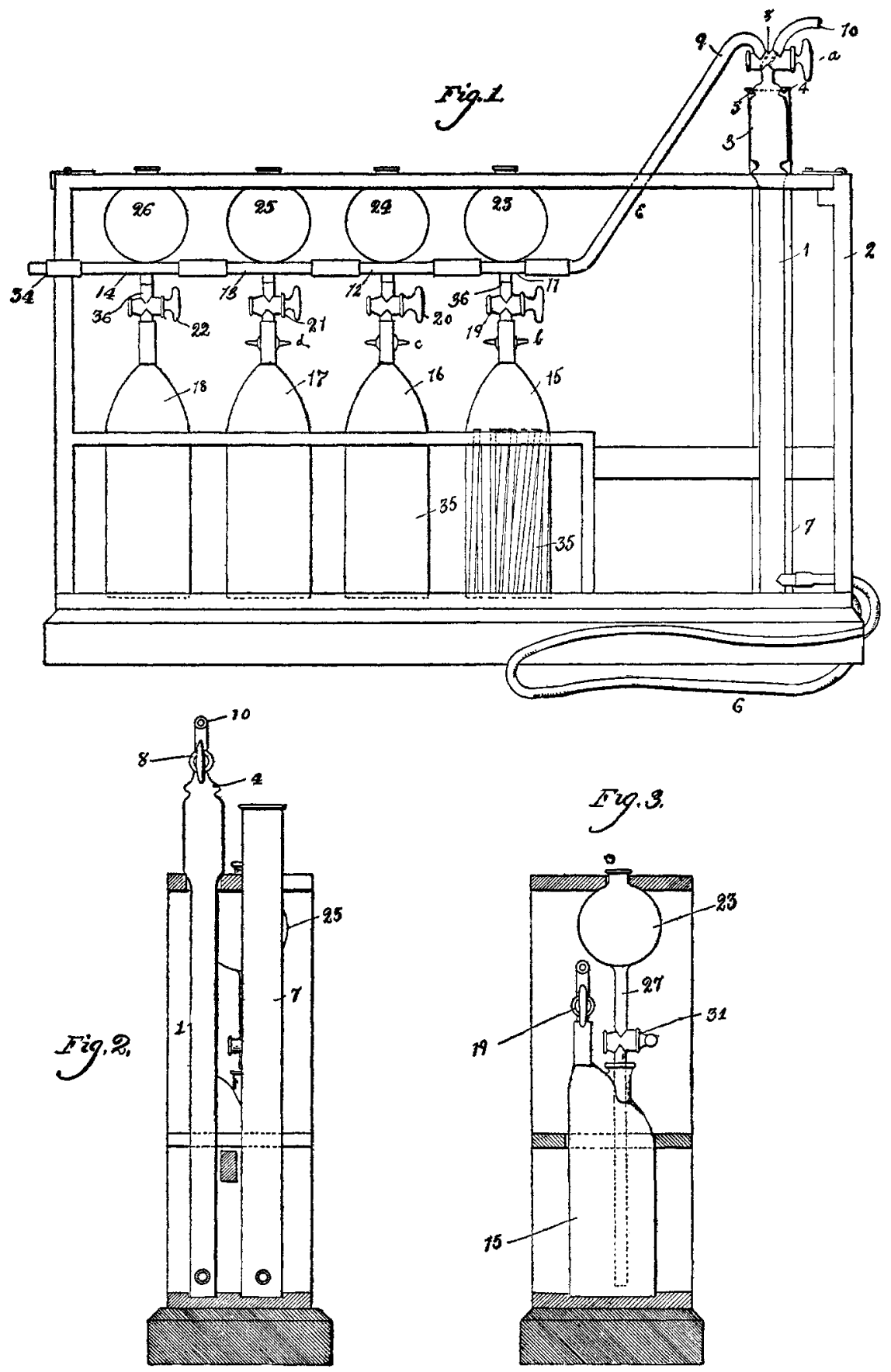


protected from the action of the light by a black cloth. Bottle I 8 is filled with water, as it is only used for storing gas.

Before beginning the analysis stop-cocks $b, c$, and $d$ are opened. Upon opening the three-way cock $a$ and raising the leveling tube 7 , the burette is filled with water, also the capillary tube Io, which, after closing $a$, is connected with the gas supply. By lowering 7 and again opening $a$, gas is drawn into the burette; Ioo cc. are measured after allowing the sides of the burette to drain. Communication is now opened between the burette and bottle I 5 by means of stop-cocks $a$ and 19 ; raising 7 forces the gas through the capillary tubing into 15 . This operation is repeated three times, always allowing the water in the burette just to enter the capillary system finally bringing the solution to the mark. Ig is now closed, and the water in the burette and tube 7 is brought to the same level before closing a. After draining, the loss in volume is observed. The same process is carried out substantially as described for the absorption of the illuminants and oxygen by bromine and phosphorus in bottles $\mathrm{I} 6$ and $I 7$ respectively, precaution being taken to completely absorb the bromine vapors in the gas after the elimination of the illuminants, by potassium liydroxide in bottle $I_{5}$ and allowing the fumes to almost disappear after the absorption of the oxygen before returning the gas to the burette. Thus the carbon dioxide illuminants and oxygen are determined, after which the remainder of the gas is stored in bottle i 8 prior to exploding portions with air, or air and oxygen. These direct absorptions are followed by the introduction of about $80 \mathrm{cc}$. of air into the burette through the tube Io. This is collected before the gas because of the coarser graduation in the bulb, very accurate measurement being required in this operation. By opening $a$ and stop-cock 22 and lowering 7 , about $\mathrm{I} 5 \mathrm{cc}$. of gas are drawn into the burette. Now 22 is closed and 2 I opened, thereby filling the capillary tubing to 8 with water from $i z$ to insure an accurate determination. Now close $a$. After craining, make a reading of the volume, and after opening communication at $a$ and 19 pass the gas and air into 15 to obtain a thorough mixing, after which it is returned to the burette, the solution being brought to the mark; 19 is closed and the tubing filled as before with water from $I 7$. Close $a$ and lower 7 to reduce the 
pressure. Clamp or preferably bend the rubber fubing and introduce into a slit in the side of the frame, thereby obtaining a perfect water seal, allowing no opportunities for loss when the mixture of gas and air is exploded.

Contact is now made between the platinum terminals and an induction coil attached to a battery, a spark produced therefrom igniting the mixture. A flame is seen suddenly to run down the burette, the explosion being very mild, due to the peculiarity in construction of the burette, carbon dioxide and water being the products of combustion. The gas is now allowed to assume the temperature of the room after the pressure on the tube is released. The contraction is observed, also the amount of carbon dioxide produced by absorption in 15 , and the excess of oxygen in 17 , the same precautions as to filling the system with water after each determination being strictly adhered to, together with the suggestion that the last time the gas is passed into ${ }^{7} 7$ for the separation of the oxygen, that water from the burette be forced through the capillary system into 17 to insure complete absorption.

From the data thus obtained, it is possible to calculate the amount of hydrogen, carbon monoxide, and methane by following the well-known formulas in Sutton's "Volumetric Analysis."

$$
\begin{aligned}
& C=1 \frac{1}{2} x+\frac{1}{2} y+2 z \quad x=A-D \quad C=\text { Contraction } \\
& A=x+y+z \quad y=\frac{3 A-2 C+D}{3} \quad A=a-n= \\
& D=y+z \quad z=\frac{2 C-3 A+2 D}{3} y=\text { Carbon monoxide } \\
& x=\text { Hydrogen } \\
& D=\text { Carbon dioxide } \\
& n=\text { Nitrogen. }
\end{aligned}
$$

$$
\begin{array}{ll}
B=\frac{1}{2} x+\frac{1}{2} y+2 z & B=\text { Oxygen consumed } \\
x=A-D & A=a-n=\text { Volume of gas }-n \\
y=\frac{3 D-2 B+A}{3} & n=\text { Nitrogen } \\
z=\frac{2 B-A}{3} & D=\text { Carbon dioxide. }
\end{array}
$$


As the excess of oxygen after the combustion is absorbed by phosphorus in bottle 77 an explosion with hydrogen is avoided. What remains after this process is completed is the nitrogen in the air plus the nitrogen in the gas; a direct determination of the latter is thus made possible.

If it should be desired to employ this apparatus for fuel gas analysis, bottles $I 7$ and 18 can be filled with a cuprous chloride solution, using a layer of petroleum in the funnels to prevent the air having an oxidizing action upon the copper solutions. By the use of two solutions a more accurate determination is made as it is well known that one cuprous chloride solution repeatedly used is not a satisfactory reagent for the absorption of carbon monoxide. It has been found that a lubricant is absolutely essential for success with this as with similar forms of apparatus. The one mentioned in this Journal, 20,678, is recommended but for the bottle containing bromine, vaseline is better adapted. A uniform temperature should be maintained in the laboratory as the burette is not water-jacketed. The apparatus described appears to have many advantageous features beside its compactness, which is certainly desirable, for ample space is allowed above the funnels for an induction coil and small battery, making the complete outfit to go in a case sixteen by seventeen inches. The $\mathrm{T}$ tubes being separate and 36 having a plug, 34 affords opportunity for rapid and easy cleansing of the capillary system. After the gas is once collected, there is no possibility of loss as it remains in the apparatus until the analysis is complete and, if necessary, severàl confirmatory explosions are made. All the parts are readily disconnected and sections when broken can be easily replaced. With the exception of $\mathrm{I} 7$, disconnection is only required in case of accident, as the other bottles may be replenished by forcing the liquids into the funnels, siphoning off and fresh solutions added. Direct explosion in the measuring burette is an advantage as it eliminates the necessity of an extra bottle for that purpose. Less than an hour is required for the completion of an analysis by this method and the results obtained have been eminently satisfactory. 